\title{
Meticillin-resistant Staphylococcus aureus CC398 is an increasing cause of disease in people with no livestock contact in Denmark, 1999 to 2011
}

J Larsen ${ }^{1}$, A Petersen ${ }^{1}$, M Sørum ${ }^{1}$, M Stegger ${ }^{1}$, L van Alphen ${ }^{1}$, P Valentiner-Branth ${ }^{2}$, LK Knudsen ${ }^{2}$, LS Larsen ${ }^{3}$, B Feingold ${ }^{4}$, LB Price ${ }^{56}$, PS Andersen ${ }^{1}$, AR Larsen ${ }^{1}$, RL Skov ${ }^{1}$

1. Microbiology and Infection Control, Statens Serum Institut, Copenhagen, Denmark

2. Infectious Disease Epidemiology, Statens Serum Institut, Copenhagen, Denmark

3. National Food Institute, Technical University of Denmark, Søborg, Denmark

4. Department of Environmental Health Sciences, University at Albany, State University of New York, School of Public Health, Rensselaer, NY, United States

5. Center for Food Microbiology and Environmental Health, Translational Genomics Research Institute, Flagstaff, AZ, United States

6. Milken Institute School of Public Health, George Washington University, Washington, DC, United States

Correspondence: Jesper Larsen (JRL@ssi.dk)

Citation style for this article:

Larsen J, Petersen A, Sørum M, Stegger M, van Alphen L, Valentiner-Branth P, Knudsen LK, Larsen LS, Feingold B, Price LB, Andersen PS, Larsen AR, Skov RL. Meticillin-resistant Staphylococcus aureus CC398 is an increasing cause of disease in people with no livestock contact in Denmark, 1999 to 2011 . Euro Surveill. 2015;20(37): pii=30021. DOI: http://dx.doi.org/10.2807/1560-7917.ES.2015.20.37.30021

Livestock constitutes a potential reservoir of meticillin-resistant Staphylococcus aureus isolates belonging to a recently derived lineage within clonal complex 398 (MRSA CC398-IIa). Since its discovery in the early 2000s, this lineage has become a major cause of human disease in Europe, posing a serious public health challenge in countries with intensive livestock production. To retrace the history of human colonisation and infection with MRSA CC398-Ila in Denmark, we conducted a nationwide, retrospective study of MRSA isolates collected from 1999 to 2011. Among 7,429 MRSA isolates screened, we identified 416 MRSA CC398-Ila isolates. Of these, 148 were from people with infections, including $\mathbf{5 1}$ from patients reporting no livestock exposure. The first cases of MRSA CC 398 -Ila infection in Denmark occurred in 2004. Subsequently, the incidence of MRSA CC 398 -lla infection showed a linear annual increase of $66 \%$ from 2004 to 2011 (from 0.09 to 1.1 per 100,000 person-years). There were clear temporal and spatial relationships between MRSA CC398-Ila-infected patients with and without livestock exposure. These findings suggest substantial dissemination of MRSA CC398-Ila from livestock or livestock workers into the Danish community and underscore the need for strategies to control its spread both on and off the farm.

\section{Introduction}

In 2005, two studies, from France and the Netherlands, provided the first evidence of a reservoir of meticillinresistant Staphylococcusaureus (MRSA) in livestock, with transmission to humans $[1,2]$. The MRSA isolates from these initial cases belonged to clonal complex
398 (CC398), which was very uncommon in humans at the time. Since its discovery, MRSA CC 398 has been isolated from cattle, horses, chickens and turkeys, but currently pigs appear to be its primary host [3]. While several other MRSA strain types have been identified in a variety of livestock species worldwide, $\mathrm{CC}_{398}$ is the dominant MRSA strain type in European livestock today [3].

MRSA CC398 has unique genetic characteristics compared with other MRSA strain types: it is nontypeable by Smal-pulsed-field gel electrophoresis (PFGE) [4], it comprises a distinct set of spa types [5], and it contains a novel Sau1 type I restriction-modification system [6]. These features challenged early genotyping efforts, which have been aided more recently by whole-genome sequencing.

Whole-genome phylogenetic analyses show that there are multiple $S$. aureus $\mathrm{CC}_{398} 8$ lineages in circulation, including one recently derived lineage primarily found in livestock, termed CC398-Ila, and several other more basal lineages primarily found in humans, collectively referred to as CC398-I/II-GOI [7]. The CC398-IIa isolates can be distinguished from the CC398-I/II-GOI isolates by lineage-specific canonical single nucleotide polymorphisms (canSNPs) [7,8]. Furthermore, CC398-Ila isolates are typically positive for the tetracycline resistance gene tet $(M)$ and negative for the staphylococcal complement inhibitor gene scn, whereas CC398-I/II-GOI isolates are typically negative for tet(M) and positive for scn $[7,8]$. Finally, CC398-Ila isolates generally lack the lukS-PV and lukF-PV genes encoding Panton-Valentine 
TABLE 1

Genotypic characterisation of meticillin-resistant

Staphylococcus aureus (MRSA) CC398 isolates, one isolate per person, Denmark, 1999-2011 $(\mathrm{n}=420)$

\begin{tabular}{|c|c|c|c|c|c|c|}
\hline \multirow{2}{*}{ Strain type } & \multirow{2}{*}{$\operatorname{tet}(M)$} & \multirow{2}{*}{ sen } & \multicolumn{3}{|c|}{ canSNP } & \multirow{2}{*}{$\begin{array}{c}\text { Number } \\
(\%)\end{array}$} \\
\hline & & & 748 & 1002 & 3737 & \\
\hline \multicolumn{7}{|l|}{ MRSA CC398-IIa } \\
\hline MRSA CC398-IIa & + & - & ND & ND & ND & 389 (94) \\
\hline MRSA CC398-IIa & + & + & $T$ & A & A & $25(6)$ \\
\hline MRSA CC398-IIa & - & - & $\mathrm{T}$ & A & A & $2(0.5)$ \\
\hline \multicolumn{6}{|l|}{ Total } & $416(100)$ \\
\hline \multicolumn{7}{|l|}{ MRSA CC398-I/II-GOI } \\
\hline MRSA CC398-I/II-GOI & - & + & ND & ND & ND & $4(100)$ \\
\hline \multicolumn{6}{|l|}{ Total } & $4(100)$ \\
\hline
\end{tabular}

canSNP: canonical single nucleotide polymorphism; ND: not determined.

+ : positive; - : negative.

leukocidin (PVL), whereas these genes are frequently found in CC398-I/II-GOI isolates [8]. The existence of two epidemiologically and evolutionary distinct $S$. aureus $\mathrm{CC}_{398} 8$ lineages underscores the importance of strain typing when undertaking epidemiological investigations and source tracking of $S$. aureus $\mathrm{CC}_{398}$.

Despite its recent emergence as a zoonotic pathogen [1-3], MRSA CC398 has become a frequent cause of human colonisation and disease in Europe, especially in countries with intensive livestock production [9]. For example, MRSA $\mathrm{CC}_{3} 98$ accounts for up to $40 \%$ of new cases of MRSA in Denmark, the Netherlands and some areas of Germany [10-12]. MRSA CC398 primarily colonises and infects people with direct livestock contact (primary exposure) and their household members through intra-household transmission (secondary exposure). However, surveillance data from Denmark and the Netherlands show that MRSA CC 398 is also found in people with no connection to livestock $[10,13]$. In addition, MRSA CC398 has been implicated in sporadic outbreaks in Dutch hospitals and nursing homes [14-16]. Unfortunately, these studies did not differentiate MRSA CC398-IIa isolates from MRSA CC398-I/II-GOI isolates, and it is therefore unclear how and to what extent MRSA CC 398 -Ila is spreading to people with no livestock exposure. S. aureus normally spreads through human-to-human contact [17] and this mode of transmission is likely to play a major role in the dissemination of MRSA CC398-Ila. Various studies suggest that environmental contamination of air and soil surfaces may also contribute to MRSA CC398 transmission [1821]. Moreover, MRSA $\mathrm{CC}_{398} 8$ is a relatively common contaminant of retail meat in Europe [22], and foodborne transmission has been hypothesised as a possible source of infections in people with no livestock contact. However, epidemiological data suggest that food-borne transmission is rare [22].
The aim of our study was to investigate the epidemiology of MRSA CC398 in humans in Denmark from 1999 to 2011, especially in relation to the presence of MRSA CC-398-Ila in people with no livestock contact.

\section{Methods}

\section{National MRSA registry and strain repository, 1999-2011}

This study is based on data from the national MRSA registry and strain repository at Statens Serum Institut in Copenhagen. It should be noted that the registry contains data available only at the time of the first diagnosis and does not hold information on subsequent episodes of infection or asymptomatic carriage. The use of data from the national MRSA registry was approved by the Danish Data Protection Agency (protocol no. 2001-14-0021).

Since 1988, one MRSA isolate from each newly identified MRSA-positive person (including patients with infection and people with asymptomatic carriage) has been forwarded to Statens Serum Institut from Danish clinical microbiology laboratories. From 1988 to 2006, this was as part of a voluntary surveillance programme and since 2007, has been part of a national mandatory programme for the management of MRSA [23]. Compliance with both programmes was about 100\% during the study period, as assessed by annual feedback between the clinical microbiology laboratories and Statens Serum Institut. From 1999 to 2006, all MRSA isolates were genotyped using Smal-PFGE [24]. In 2007, Smal-PFGE was replaced by PCR-based detection of mecA and lukF-PV (an indicator of PVL production) and spa typing [25].

All MRSA isolates were tested for antimicrobial susceptibility (erythromycin, clindamycin, tetracycline, fusidic acid, rifampicin, norfloxacin, kanamycin, linezolid and mupirocin) by use of the disk diffusion method, in accordance with the European Committee on Antimicrobial Susceptibility Testing guidelines [26]. Screening for reduced susceptibility to glycopeptides was performed on brain-heart infusion agar supplemented with $5 \mu \mathrm{g} / \mathrm{mL}$ teicoplanin. MRSA isolates growing on the screening agar were further tested using Etest glycopeptide-resistance detection strips (0.5-32 $\mu \mathrm{g} / \mathrm{mL}$ vancomycin, $0.5-32 \mu \mathrm{g} / \mathrm{mL}$ teicoplanin) (bioMérieux, Marcy l'Etoil, France), as described by Fitzgibbon et al. [27]. Multidrug resistance was defined as resistance to three or more non- $\beta$-lactam antibiotics.

\section{Identification and characterisation of MRSA CC398 isolates}

MRSA isolates were tentatively designated as $\mathrm{CC}_{398} 8$ if they were nontypeable by Smal-PFGE (1999-2006) or if they displayed spa types previously associated with S. aureus $\mathrm{CC}_{398}$ (2007-2011). Putative MRSA CC 398 isolates were confirmed by PCR detection of the sau1hsdS1 variant in S. aureus $\mathrm{CC}_{398}$ [6]. All MRSA CC398 isolates were assessed for the presence of tet $(\mathrm{M})$ and 


\section{TABLE 2}

Characteristics of meticillin-resistant Staphylococcus aureus (MRSA) CC398-IIa isolates and infected patients, Denmark, 1999-2011 ( $\mathrm{n}=148)$

\begin{tabular}{|c|c|c|c|}
\hline \multirow[b]{2}{*}{ Characteristic } & \multicolumn{2}{|c|}{$\begin{array}{c}\text { Number (\%) of MRSA } \\
\mathrm{CC}_{398} \text {-lla-infected patients, }\end{array}$} & \multirow[b]{2}{*}{ P value ${ }^{b}$} \\
\hline & $\begin{array}{l}\text { Livestock- } \\
\text { exposed } \\
(\mathrm{n}=97)\end{array}$ & $\begin{array}{l}\text { Livestock- } \\
\text { unexposed } \\
(\mathrm{n}=51)\end{array}$ & \\
\hline \multicolumn{4}{|l|}{ spa type } \\
\hline to11 & $10(10)$ & $6(12)$ & 0.79 \\
\hline to34 & $83(86)$ & $39(76)$ & 0.18 \\
\hline $\mathrm{t} 108$ & o (o) & $2(4)$ & 0.12 \\
\hline$t_{571}$ & $1(1)$ & o (o) & 1.00 \\
\hline t899 & o (o) & $1(2)$ & 0.34 \\
\hline $\mathrm{t} 1255$ & $1(1)$ & o (o) & 1.00 \\
\hline $\mathrm{t} 1446$ & o (o) & o (o) & NA \\
\hline t1793 & o (o) & o (o) & NA \\
\hline t5095 & o (o) & $2(4)$ & 0.12 \\
\hline $\mathrm{t} 5706$ & $1(1)$ & o (o) & 1.00 \\
\hline t9345 & $1(1)$ & o (o) & 1.00 \\
\hline t9517 & o (o) & $1(2)$ & 0.34 \\
\hline \multicolumn{4}{|l|}{ Presence of genes } \\
\hline $\operatorname{tet}(M)$ & $97(100)$ & $51(100)$ & NA \\
\hline$s c n$ & $3(3)$ & $3(6)$ & 0.42 \\
\hline lukF-PV & o (o) & o (o) & NA \\
\hline \multicolumn{4}{|c|}{ Antimicrobial resistance } \\
\hline Erythromycin & $40(41)$ & $16(31)$ & 0.29 \\
\hline Clindamycin & $74(76)$ & $34(67)$ & 0.24 \\
\hline Tetracycline & $97(100)$ & $51(100)$ & NA \\
\hline Fusidic acid & $1(1)$ & $3(6)$ & 0.12 \\
\hline Rifampicin & $1(1)$ & o (o) & 1.00 \\
\hline Norfloxacin & $21(22)$ & $10(20)$ & 0.83 \\
\hline Kanamycin & $7(7)$ & $6(12)$ & 0.37 \\
\hline Linezolid & o (o) & $\mathrm{o}(\mathrm{o})$ & NA \\
\hline Mupirocin & o (o) & o (o) & NA \\
\hline Glycopeptides & o (o) & $\mathrm{o}(\mathrm{o})$ & NA \\
\hline Multidrug resistance & $51(53)$ & $23(45)$ & 0.49 \\
\hline \multicolumn{4}{|l|}{ Age and sex } \\
\hline Female-to-male ratio & 0.5 & 1.3 & $0.0081^{*}$ \\
\hline $\begin{array}{l}\text { Median age in years } \\
\text { (range) }\end{array}$ & $30(0-89)$ & $49(1-84)$ & $0.0007^{*}$ \\
\hline \multicolumn{4}{|l|}{ Type of infection } \\
\hline SSTIS & $82(85)$ & $44(86)$ & 1.00 \\
\hline Ear & $8(8)$ & $3(6)$ & 0.75 \\
\hline Eye & $2(2)$ & o (o) & 0.55 \\
\hline Respiratory sites & 3(3) & $4(8)$ & 0.23 \\
\hline Bone and joint & $2(2)$ & o (o) & 0.55 \\
\hline Blood and CSF & o (o) & o (o) & NA \\
\hline Other & o (o) & o (o) & NA \\
\hline
\end{tabular}

CSF: cerebrospinal fluid; NA: not applicable; SSTIs: skin and soft tissue infections.

a Unless otherwise specified.

${ }^{*} P$ value $<0.05$ (significance level set at $a<0.05$ ).

\section{FIGURE 1}

Annual number of meticillin-resistant Staphylococcus aureus (MRSA) CC398-IIa isolates, from each newly identified MRSA-positive person, Denmark, 1999-2011 (n $=416$ )

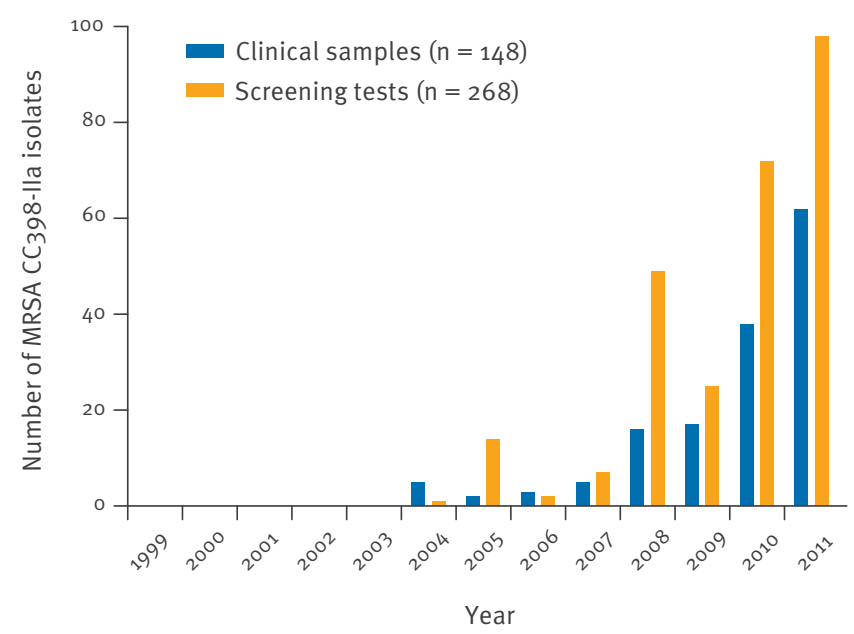

scn using a multiplex PCR assay [8]. For comparative purposes, MRSA CC398 isolates from 1999 to 2006 were subjected to PCR-based detection of mecA and lukF-PV and spa typing [25]. MRSA CC398-Ila isolates were differentiated from other $\mathrm{CC}_{39} 8$ lineages using a dual-probe real-time PCR assay targeting CC398-Ilaspecific canSNPs $[7,8]$.

\section{Clinical and epidemiological investigations}

Patient information (i.e. age, sex, residential address, medical history and known livestock contact) was obtained from hospital or general practice records from 1999 to 2006 and from notification forms since 2007 , when MRSA became a notifiable organism in Denmark. Patient information, including livestock contact or not, that was insufficiently described in the written records was obtained retrospectively through interviews with the corresponding patients, the relevant hospital or their general practitioner.

MRSA CC398 infections in patients who reported direct livestock contact (primary exposure) or were living together with a person with direct livestock contact (secondary exposure) were classified as livestockonset. MRSA CC398 infections in patients with no livestock contact were classified either as communityonset when the positive culture was obtained from an outpatient or within the first 48 hours of hospital admission, or as healthcare-onset when the positive culture was obtained from an inpatient after 48 hours of hospital admission.

Infections were grouped as: (i) skin and soft tissue infections (SSTIs), including nose, skin, wound, and abscess; (ii) ear; (iii) eye; (iv) respiratory sites, including tracheal aspirates, sputum and induced sputum; (v) bone and joint; (vi) blood and cerebrospinal fluid; and (vii) others, including all other clinical sites, such as indwelling devices and fluid of unspecified origin. 


\section{FIGURE 2}

Annual number (A) and scatter plot (B) of meticillinresistant Staphylococcus aureus (MRSA) CC398-IIa infections, Denmark, 2004-2011 ( $\mathrm{n}=148)$

A.

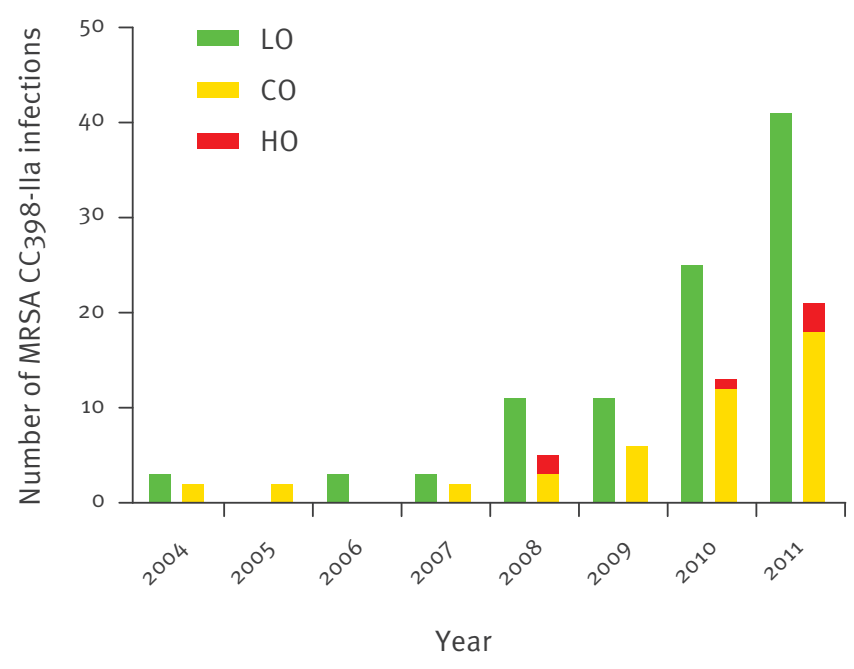

B.

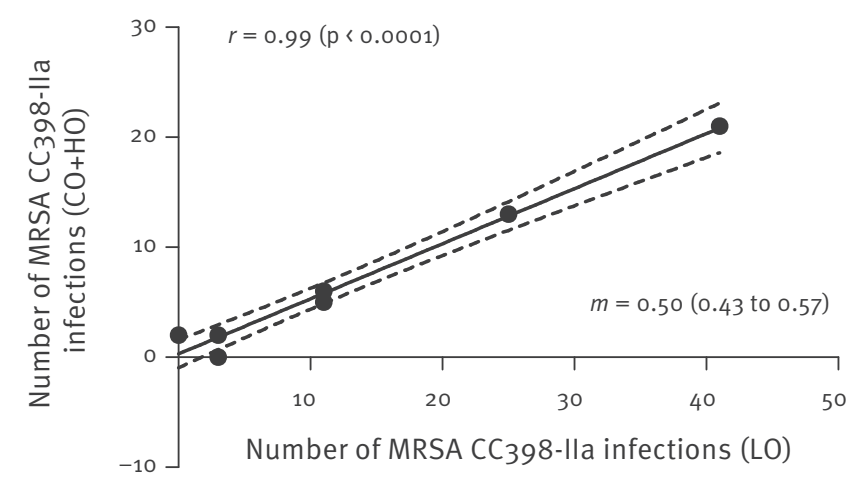

CO: community-onset disease; HO: healthcare-onset disease; LO: livestock-onset disease.

Pearson's correlation ( $r$ ) with $p$ values in parentheses, linear regression line (solid) and $95 \%$ confidence intervals (dashed lines) and slope $(\mathrm{m})$ with $95 \%$ confidence intervals in parentheses are shown.

\section{Temporal and spatial analyses of MRSA} CC398-IIa infections

We assessed the temporal and spatial relationships between MRSA CC398-Ila-infected patients with and without livestock exposure (healthy carriers identified through screening tests were excluded from epidemiological analyses, to eliminate any bias or confounding due to inconsistent screening practices). Discrete and continuous variables were compared between the two exposure groups by use of Fisher's exact test and Student's t-test, respectively (GraphPad Prism software, version 5, GraphPad, La Jolla, California, United States). Poisson regression modelling was used to compare incidence over time and between the two exposure groups (Stata software, version 12, StataCorp, College Station, Texas, United States). Pearson correlation and linear regression were used to describe the strength of the linear relationship between annual numbers of infections among patients with and without livestock exposure (GraphPad Prism software, version 5, GraphPad, La Jolla, California, United States). The significance level was set at $a=0.05$.

To characterise the spatial distribution of patients, we plotted their georeferenced residential addresses as point data on digital maps along with the population density per $\mathrm{km}^{2}$ for each municipality (ArcGIS software, version 10.1, ESRI, Redlands, California, United States). Each data point was placed randomly within a $5-\mathrm{km}$ radius of the exact residential address within a given municipality of residence to protect anonymity of the patient. Data on the number of person-years and the population density per $\mathrm{km}^{2}$ for each municipality were obtained from Statistics Denmark.

\section{Results}

Identification of MRSA CC398 isolates, spa typing and antimicrobial susceptibilities

Statens Serum Institut received 7,429 MRSA isolates, one isolate per person, over the 13-year study period from 1999 to 2011. A total of 420 isolates were identified as MRSA CC 398 , of which 416 putatively belonged to $\mathrm{CC}_{398}$-Ila based on the presence/absence of tet $(\mathrm{M})$ and $\operatorname{scn}(n=389)$ or detection of canSNPs $(n=27)$ (Table 1). The remaining four isolates belonged to CC398-I/II-GOI.

The 416 MRSA CC 398 -Ila isolates displayed 12 different spa types, including to34 $(\mathrm{n}=343 ; 82 \%)$, to11 $(\mathrm{n}=45$; $11 \%), \mathrm{t} 5706(\mathrm{n}=8 ; 1.9 \%), \mathrm{t} 571(\mathrm{n}=6 ; 1.4 \%), \mathrm{t} 108(\mathrm{n}=3$; $0.7 \%), \mathrm{t} 5095$ ( $n=3 ; 0.7 \%), \mathrm{t} 1255(\mathrm{n}=2 ; 0.5 \%), \mathrm{t} 9517$ $(n=2 ; 0.5 \%), t 899(n=1 ; 0.2 \%), t 1446(n=1 ; 0.2 \%)$, t1793 $(n=1 ; 0.2 \%)$ and t9345 $(n=1 ; 0.2 \%)$, were negative for lukF-PV and demonstrated variable levels of antimicrobial resistance: tetracycline $(n=416 ; 100 \%)$, clindamycin ( $n=309 ; 74 \%)$, erythromycin $(n=188$; $45 \%)$, norfloxacin $(n=91 ; 22 \%)$, kanamycin $(n=26$; $6.3 \%$ ), fusidic acid ( $n=7 ; 1.7 \%)$, rifampicin ( $n=2$; $0.5 \%$ ), linezolid $(n=1 ; 0.2 \%)$. None were resistant to mupirocin or glycopeptides. Most of the MRSA CC 398 Ila isolates $(n=237 ; 57 \%)$ were multidrug resistant.

All four MRSA CC398-I/II-GOI isolates displayed spa type to34, were positive for lukF-PV and were resistant to erythromycin, clindamycin and norfloxacin.

\section{Clinical epidemiology}

A total of 151 MRSA CC 398 isolates were obtained from clinical cases: the remaining 269 MRSA CC 398 isolates originated from screening tests and were excluded from further analysis. Among the 151 cases of MRSA CC398 infection, 97 had primary or secondary exposure to livestock and 54 had no livestock exposure. MRSA CC398-Ila accounted for all 97 infections in patients with primary or secondary exposure to livestock and for 51 of the 54 infections in patients with 


\section{FIGURE 3}

Geographical distribution of patients with meticillin-resistant Staphylococcus aureus (MRSA) CC398-IIa infection, Denmark, 2004-2011 $(\mathrm{n}=148)$

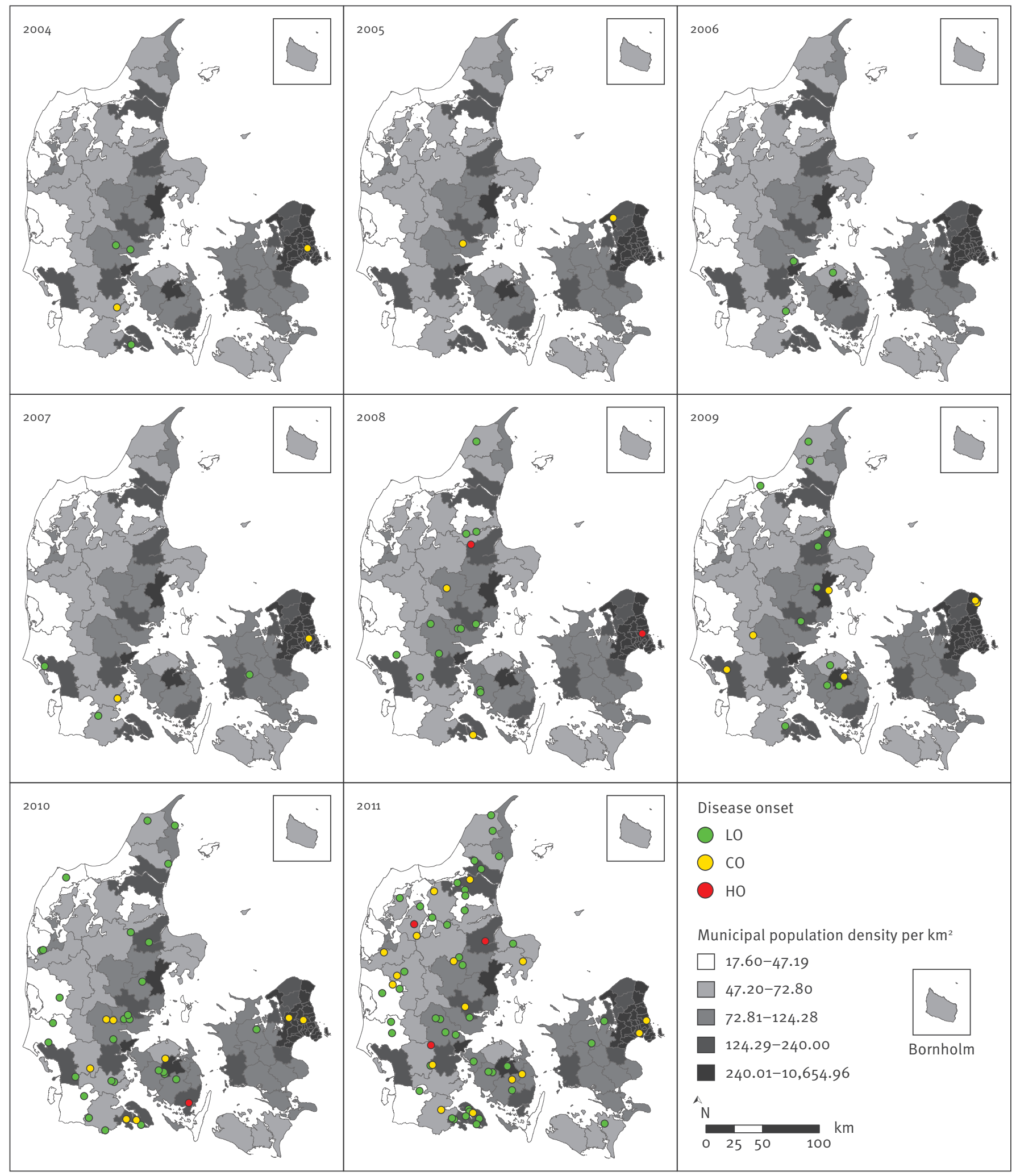

CO: community-onset disease; HO, healthcare-onset disease; LO: livestock-onset disease.

Each dot is placed randomly within a $5-\mathrm{km}$ radius of the exact residential address within a given municipality of residence to protect anonymity of the patient. The municipal population density per $\mathrm{km}^{2}$ is shown. 
no livestock exposure, including 45 cases with community-onset infection and six cases with healthcareonset infections. MRSA CC $398-1 / 11-G O I$ was identified in the remaining three patients with no livestock contact, including two adoptees from Asia and a close family member of one of the adoptees, all of whom had SSTIs.

MRSA CC398-Ila isolates from patients unexposed to livestock were highly similar to MRSA CC398-Ila isolates from livestock-exposed patients both in terms of molecular and phenotypic characteristics as well as type of infection (Table 2). Unexposed patients were significantly older than livestock-exposed patients (median age: 49 vs 30 years; $p=0.0007$ ) and were more likely to be female (female-to-male ratio: 1.3 vs $0.5 ; p=0.0081$ ) (Table 2).

\section{Temporal and spatial trends of MRSA CC398-}

\section{IIa infections}

The annual numbers of MRSA CC 398 -Ila isolates, from each newly identified MRSA-positive person, over the 13-year study period from 1999 to 2011 are shown in Figure 1. MRSA CC398-lla was first identified in January 2004 in a patient with an SSTI. Subsequently, the incidence of MRSA CC 398 -lla infections increased from 0.09 per 100,000 person-years in 2004 to 1.1 per 100,000 person-years in 2011 , corresponding to a linear annual increase of $66 \%$ (incidence rate ratio (IRR): 1.7; 95\% confidence interval (CI): 1.5-1.9; $p$ < 0.00001 ). Pearson correlation and linear regression demonstrated a clear temporal relationship between annual number of infections among livestock-exposed and unexposed patients (Figure 2). Furthermore, most unexposed patients appeared to live in close proximity to livestock-exposed patients (Figure 3).

\section{Incidence of MRSA CC398-IIa infection among} people with no livestock contact

In 2011, a total of 62 cases of MRSA CC398-Ila infection were identified in Denmark (5,475,791 inhabitants), of which 66\% (41/62) were livestock-exposed and 34\% (21/62) were unexposed (see also Figure 2 and Figure 3). The 41 livestock-exposed patients lived in 25 of the 99 Danish municipalities. In these 25 municipalities ( $1,676,186$ inhabitants), the incidence of MRSA CC 398 Ila infections among unexposed people was 0.7 per 100,000 person-years, whereas the overall incidence of MRSA infections was 10.9 per 100,000 person-years. In the remaining 74 municipalities $(3,799,605$ inhabitants), the incidence of MRSA CC398-lla infections among unexposed people was 0.3 per 100,000 personyears, whereas the overall incidence of MRSA infections was 12.8 per 100,000 person-years. Poisson regression modelling showed that the risk of MRSA CC398-Ila infection among unexposed people was significantly higher in the 25 municipalities in which livestockexposed patients lived than in the rest of Denmark (74 municipalities) (IRR: $2.5 ; 95 \% \mathrm{Cl}: 1.1$ to $5.7 ; \mathrm{p}=0.041$ ). Nonetheless, MRSA CC398-Ila accounted for only 6\% $(11 / 183)$ and $2 \%(10 / 487)$ of the total number of MRSA infections among unexposed people in the 25 and 74 municipalities, respectively, and the overall risk of MRSA infection was not significantly different between the two groups of municipalities (IRR: 0.9; 95\% Cl: 0.7 to $1.0 ; p=0.062$ ).

In the Capital Region (30 municipalities, 1,645,825 inhabitants), we observed only two cases of MRSA CC398-Ila infection among unexposed people in 2011, corresponding to $0.6 \%(2 / 309)$ of the total number of MRSA infections among unexposed people.

\section{Discussion}

The results presented here show that MRSA CC398-Ila was an increasing cause of infection among people with and without livestock exposure in Denmark from 2004 to 2011. During this time, there was a more than fourfold increase in the prevalence of MRSA $\mathrm{CC}_{39} 8$ among Danish pigs $[5,28,29]$. Most of the unexposed patients were spatially clustered around livestockexposed patients. Moreover, MRSA CC 398 -Ila isolates from livestock-exposed and unexposed patients had similar molecular and phenotypic characteristics. Together, these findings suggest that the expanding livestock reservoir of MRSA $\mathrm{CC}_{398} 8$ may have led to increased spillover into the surrounding community during the study period.

The MRSA CC398-Ila isolates analysed demonstrated high levels of resistance to tetracyclines, lincosamides (clindamycin), macrolides (erythromycin) and quinolones (norfloxacin), which, with the exception of quinolones, represent some of the most commonly used antibiotics in Danish pig production [29]. Interestingly, the isolates were more often resistant to clindamycin than to erythromycin. This rather unusual resistance pattern has been described in human and porcine MRSA CC398 isolates from Spain, where it was associated with the presence of either the $\ln u(A)$ or $\ln u(B)$ gene [30]. Most of the MRSA CC398-Ila isolates were multidrug resistant, thus further limiting the options for clinical therapy beyond $\beta$-lactam antibiotics. In Denmark, use of antibiotics for growth promotion and prophylaxis is not permitted in livestock production; therefore, the data presented here suggest that the public health risks of antibiotic use in agriculture may also include therapeutic applications.

While the MRSA CC398-Ila isolates and types of infection did not differ among the two exposure groups, the patients comprising the two populations varied significantly. Livestock-exposed patients were younger than unexposed patients and were more likely to be male. These findings probably reflect the demographics of livestock workers, who are more likely to be workingage men compared with the general population.

MRSA CC 398-Ila was primarily associated with SSTIs and other non-invasive infections during the study period; however, there have been four fatal bloodstream infections (BSIs) with MRSA CC398-Ila in Denmark since 2012 [31]. These patients had no livestock exposure 
but presented with known predisposing risk factors of MRSA BSI (e.g. severe underlying diseases or haemodialysis). These observations confirm that MRSA CC398Ila is capable of causing life-threatening disease in at-risk patients, and it is expected that there will be an increasing number of invasive infections in the near future if the prevalence of MRSA CC398-Ila continues to increase in the general population. Thus, there is an urgent need to control this organism in the healthcare setting. In 2012, the Danish Health and Medicines Authority released updated guidelines for the management of MRSA, which recommend that all hospitals perform targeted screening of patients and hospital staff at high risk of MRSA CC398-Ila carriage (i.e. all people with primary or secondary exposure to livestock) [23]. However, spread into the hospital may increase if the prevalence of MRSA CC398-Ila in the unexposed population continues to grow.

Our study does not address how MRSA CC398-Ila spreads from the livestock reservoir into the local community. Other $S$. aureus strain types predominantly spread through human-to-human contact [17]. The same is likely to be true for MRSA CC398-Ila, where livestock workers may be the primary source for the delivery of MRSA CC398-Ila to unexposed people. In addition, some unique features of livestock production may make it more conducive to environmental transmission. For example, powerful tunnel ventilation systems that carry air though livestock production facilities may mediate airborne transmission of MRSA CC398-Ila into nearby communities. Previous studies have shown that antibiotic-resistant $S$. aureus can be isolated from air samples up to $300 \mathrm{~m}$ downwind of pig farms $[18,19]$. Likewise, land application of pig manure as fertiliser, by spray or injection, may also mediate environmental transmission. Two studies from the United States showed significant associations between the cropland application of pig manure and MRSA carriage [20,21]. However, in contrast to our study, these two American studies did not distinguish between people with and without livestock contact and did not report MRSA strain types. More research is needed to quantify the relative roles of human contact and environmental media in the transmission of MRSA CC398-Ila to people with no livestock exposure.

MRSA CC398-Ila is frequently isolated from retail meat in Denmark $[28,29,30,32]$, which has raised concerns about food-borne acquisition of this pathogen in both community and healthcare settings through consumption or handling of contaminated products. These concerns were substantiated by a case-control study from the Netherlands, which found that consumption of chicken meat was a significant risk factor for MRSA carriage [33]. In Denmark, most livestock is slaughtered in a small number of centralised abattoirs, from which meat is distributed to widely dispersed retail stores. Despite this, most cases of MRSA CC398-Ila infection in our study were among unexposed people who lived in close proximity to livestock-exposed patients in rural areas; we observed only a few cases of MRSA CC398-Ila infection among unexposed people in the Capital Region. Taken together, these findings strongly suggest that food-borne transmission does not play a major role in the MRSA CC398-Ila epidemiology.

The low prevalence of MRSA CC398-I/II-GOI in our study suggests that this strain type is not endemic in Denmark. In contrast, MRSA CC398-I/II-GOI is a frequent cause of both community-onset and healthcareonset infections in China [34] and has been described in Asian adoptees in the Netherlands and Sweden $[35,36]$. Consistent with these previous studies, the patients with MRSA CC398-I/II-GOI infection identified in our study were linked to adoptees from Asia.

Our study has several strengths. First, we used a nationwide registry-based case-finding strategy in combination with individual-level information on both primary and secondary exposure to livestock. Second, we eliminated any bias or confounding due to inconsistent screening practices by excluding healthy carriers identified through screening tests from the analysis. Third, we used a set of newly developed genotyping tools to differentiate between MRSA CC398-Ila and MRSA CC398-I/II-GOI isolates.

Our study also has limitations that should be considered. First, the national MRSA registry contains data only at the time of the first diagnosis and does not hold information on subsequent episodes of infection. By discounting people with asymptomatic carriage who later develop MRSA infections, the number of MRSA infections may be substantially underestimated. Second, it is unclear if our findings are generalisable to other countries. Nonetheless, our findings are consistent with those from the Netherlands, where MRSA CC398-positive people with no livestock exposure were found to be concentrated in rural, livestock-production areas [37].

Our findings suggest that MRSA CC398-Ila may be spreading from the livestock reservoir into the local community with increasing frequency. However, even in these local communities, MRSA CC398-Ila accounted for a relatively small proportion of the total number of MRSA infections among unexposed people, and there was no apparent association between living inside or outside such an area and the overall risk of acquiring an MRSA infection. The spatial distribution and rate of spillover of MRSA CC398-Ila into the community are likely to increase in the near future as the livestock reservoir expands to MRSA-naive farms. The epidemiology of MRSA CC398-Ila may also change over time as new variant subclones emerge with increased or decreased capacity for human-to-human transmission. Therefore, it is important to continue monitoring MRSA CC398-Ila colonisation and infection rates among livestock and livestock workers, community dwellers and patients and staff in healthcare facilities. Efforts must also be 
made to further resolve transmission routes and stem the continued dissemination of MRSA CC398-IIa.

\section{Acknowledgements}

We thank the Danish clinical microbiology laboratories for making this study possible and Stine Frese-Madsen, Lone Ryste Kildevang Hansen and Julie Hindsberg Nielsen for molecular and phenotypic characterisation of MRSA. The present study was supported by the National Institute of Allergy and Infectious Diseases, National Institutes of Health (project number 1R01Al101371-01A1).

\section{Conflict of interest}

None declared.

\section{Authors' contributions}

$J L, A P, M S, M S, A L$ and RS were members of the MRSA surveillance team. JL, $L P, P A, A L$ and $R L$ designed the study. JL, $L P$ and RS prepared the initial manuscript. AP and $A L$ contributed to the subsequent editorial revisions. LvA, PVB, LK, LL and $B F$ performed epidemiological investigations.

\section{References}

1. Armand-LefevreL, RuimyR, AndremontA. Clonal comparison of Staphylococcus aureus isolates from healthy pig farmers, human controls, and pigs.Emerg Infect Dis. 2005;11(5):711-4. DOI: 10.3201/eid1105.040866 PMID: 15890125

2. VossA, LoeffenF, BakkerJ, KlaassenC, WulfM. Methicillinresistant Staphylococcus aureus in pig farming. Emerg Infect Dis. 2005;11(12):1965-6. DOI: 10.3201/eid1112.050428 PMID: 16485492

3. FitzgeraldJR. Livestock-associated Staphylococcus aureus: origin, evolution and public health threat.Trends Microbiol. 2012;20(4):192-8. DOI: 10.1016/j.tim.2012.01.006 PMID: 22386364

4. BensCC, VossA, KlaassenCHJ. Presence of a novel DNA methylation enzyme in methicillin-resistant Staphylococcus aureus isolates associated with pig farming leads to uninterpretable results in standard pulsed-field gel electrophoresis analysis.J Clin Microbiol. 2006;44(5):1875-6. DOI: $10.1128 / J C M .44 \cdot 5 \cdot 1875-1876.2006$ PMID: 16672428

5. European Food Safety Authority (EFSA). Analysis of the baseline survey on the prevalence of methicillin-resistant Staphylococcus aureus (MRSA) in holdings with breeding pigs, in the EU, 2008. Part A: MRSA prevalence estimates. EFSA Journal. 2009;7(11):1376. Available from: http://www.efsa. europa.eu/sites/default/files/scientific_output/files/main documents $/ 1376 \% 2$ Co.pdf

6. SteggerM, LindsayJA, MoodleyA, SkovR, BroensEM, GuardabassiL. Rapid PCR detection of Staphylococcus aureus clonal complex 398 by targeting the restriction-modification system carrying sau1-hsdS1.J Clin Microbiol. 2011;49(2):732-4. DOI: 10.1128/JCM.01970-10 PMID: 21123532

7. PriceLB, SteggerM, HasmanH, AzizM, LarsenJ, AndersenPS, et al. Staphylococcus aureus CC398: host adaptation and emergence of methicillin resistance in livestock. MBio. 2012;3(1):e00305-11. DOI: 10.1128/mBio.00305-11 PMID: 22354957

8. SteggerM, LiuCM, LarsenJ, SoldanovaK, AzizM, ContenteCuomoT, et al. Rapid differentiation between livestockassociated and livestock-independent Staphylococcus aureus CC398 clades. PLoS ONE. 2013;8(11):e79645. DOI: 10.1371/ journal.pone.0079645 PMID: 24244535

9. van CleefBA, MonnetDL, VossA, KrziwanekK, AllerbergerF, StruelensM, et al. Livestock-associated methicillin-resistant Staphylococcus aureus in humans, Europe. Emerg Infect Dis. 2011;17(3):502-5. DOI: 10.3201/eid1703.101036 PMID: 21392444

10. DANMAP 2012. Use of antimicrobial agents and occurrence of antimicrobial resistance in bacteria from food animals, food and humans in Denmark. Copenhagen: Statens Serum Institut, National Veterinary Institute and National Food Institute,
Technical University of Denmark; September 2013. [Accessed 30 Oct 2014]. Available from: http://www.danmap.org/ Downloads/ /media/Projekt\%20sites/Danmap/DANMAP\%20 reports/DANMAP\%202012/Danmap_2012.ashx

11. NETHMAP 2013: consumption of antimicrobial agents and antimicrobial resistance among medically important bacteria in the Netherlands. Nijmegen and Bilthoven: Dutch Working Party on Antibiotic Policy and Centre for Infectious Disease Control, National Institute for Public Health and the Environment of the Netherlands; 2013. [Accessed 30 Oct 2014]. Available from: http://www.swab.nl/swab/cms3.nsf/uploads/ADFB2606CCFDF 6E4C1257BDBo022F93F/\$FILE/Nethmap_2013\%20def_web.pdf

12. KöckR, SchaumburgF, MellmannA, KöksalM, JurkeA, Beckerk, et al. Livestock-associated methicillin-resistant Staphylococcus aureus (MRSA) as causes of human infection and colonization in Germany. PLoS ONE. 2013;8(2):e55040. DOI: 10.1371/journal.pone.0055040 PMID: 23418434

13. LekkerkerkWS, van de Sande-BruinsmaN, van der SandeMA, Tjon-A-TsienA, GroenheideA, HaenenA, et al. Emergence of MRSA of unknown origin in the Netherlands. Clin Microbiol Infect. 2012;18(7):656-61. DOI: 10.1111/j.14690691.2011.03662.x PMID: 21967090

14. WulfMW, MarkesteinA, van der LindenFT, VossA, KlaassenC, VerduinCM. First outbreak of methicillin-resistant Staphylococcus aureus ST398 in a Dutch hospital, June 2007. Euro Surveill. 2008;13(9). pii: 8051.PMID: 18445406

15. FanoyE, HelmhoutLC, van der VaartWL, WeijdemaK, van Santen-VerheuvelMG, ThijsenSF, et al. An outbreak of nontypeable MRSA within a residential care facility. Euro Surveill. 2009;14(1). pii: 19080.PMID: 19161710

16. VerkadeE, BoschT, HendriksY, KluytmansJ. Outbreak of methicillin-resistant Staphylococcus aureus ST398 in a Dutch nursing home.Infect Control Hosp Epidemiol. 2012;33(6):624-6. DOI: $10.1086 / 665726$ PMID: 22561720

17. DavidMZ, DaumRS. Community-associated methicillinresistant Staphylococcus aureus: epidemiology and clinical consequences of an emerging epidemic.Clin Microbiol Rev. 2010;23(3):616-87. DOI: 10.1128/CMR.00081-09 PMID: 20610826

18. GibbsSG, GreenCF, TarwaterPM, MotaLC, MenaKD, ScarpinoPV. Isolation of antibiotic-resistant bacteria from the air plume downwind of a swine confined or concentrated animal feeding operation.Environ Health Perspect. 2006;114(7):1032-7. DOI: 10.1289/ehp.8910 PMID: 16835055

19. SchulzJ, FrieseA, KleesS, TenhagenBA, FetschA, RöslerU, et al. Longitudinal study of the contamination of air and of soil surfaces in the vicinity of pig barns by livestock-associated methicillin-resistant Staphylococcus aureus. Appl Environ Microbiol. 2012;78(16):5666-71. DOI: 10.1128/AEM.00550-12 PMID: 22685139

20. CaseyJA, CurrieroFC, CosgroveSE, NachmanKE, SchwartzBS High-density livestock operations, crop field application of manure, and risk of community-associated methicillinresistant Staphylococcus aureus infection in Pennsylvania. JAMA Intern Med. 2013;173(21):1980-90. DOI: 10.1001/ jamainternmed.2013.10408 PMID: 24043228

21. CarrelM, SchweizerML, SarrazinMV, SmithTC, PerencevichEN. Residential proximity to large numbers of swine in feeding operations is associated with increased risk of methicillinresistant Staphylococcus aureus colonization at time of hospital admission in rural lowa veterans. Infect Control Hosp Epidemiol. 2014;35(2):190-3. DOI: 10.1086/674860 PMID: 24442084

22. WendlandtS, SchwarzS, SilleyP. Methicillin-resistant Staphylococcus aureus: a food-borne pathogen?Annu Rev Food Sci Technol. 2013;4(1):117-39. DOI: 10.1146/annurevfood-030212-182653 PMID: 23190141

23. Danish Health and Medicines Authority. Vejledning om forebyggelse af spredning af MRSA. [Guideline on the prevention of spread of MRSA]. Copenhagen: Danish Health and Medicines Authority; 2012. Danish. Available from: http:// sundhedsstyrelsen.dk/publ/Publ2012/11nov/MRSAvejl2udg. pdf

24. MurchanS, KaufmannME, DeplanoA, de RyckR, StruelensM, ZinnCE, et al. Harmonization of pulsed-field gel electrophoresis protocols for epidemiological typing of strains of methicillin-resistant Staphylococcus aureus: a single approach developed by consensus in 10 European laboratories and its application for tracing the spread of related strains. J Clin Microbiol. 2003;41(4):1574-85. DOI: 10.1128/ JCM.41.4.1574-1585.2003 PMID: 12682148

25. LarsenAR, SteggerM, SørumM. spa typing directly from a mecA, spa and pvl multiplex PCR assay-a cost-effective improvement for methicillin-resistant Staphylococcus aureus surveillance.Clin Microbiol Infect. 2008;14(6):611-4. DOI: 10.1111/j.1469-0691.2008.01995.x PMID: 18393997 
26. European Committee on Antimicrobial Susceptibility Testing (EUCAST). Breakpoint tables for interpretation of MICs and zone diameters. Version 4.0. 2014. EUCAST. [Accessed 30 Oct 2014]. Available from: http://www.eucast.org/ fileadmin/src/media/PDFs/EUCAST_files/Breakpoint_tables/ Breakpoint_table_v_4.0.pdf

27. FitzgibbonMM, RossneyAS, O’ConnellB. Investigation of reduced susceptibility to glycopeptides among methicillinresistant Staphylococcus aureus isolates from patients in Ireland and evaluation of agar screening methods for detection of heterogeneously glycopeptide-intermediate S. aureus.J Clin Microbiol. 2007;45(10):3263-9. DOI: 10.1128/JCM.00836-07 PMID: 17687008

28. DANMAP 2010. Use of antimicrobial agents and occurrence of antimicrobial resistance in bacteria from food animals, food and humans in Denmark. Copenhagen: Statens Serum Institut, Danish Medicines Agency, National Veterinary Institute and National Food Institute, Technical University of Denmark; August 2011. [Accessed 30 Oct 2014]. Available from: http:// danmap.org/ /media/Projekt\%20sites/Danmap/DANMAP\%20 reports/Danmap_2010.ashx

29. DANMAP 2011. Use of antimicrobial agents and occurrence of antimicrobial resistance in bacteria from food animals, food and humans in Denmark. Copenhagen: Statens Serum Institut, National Veterinary Institute and National Food Institute, Technical University of Denmark; September 2012. [Accessed 30 Oct 2014]. Available from: http://www.danmap.org/ Downloads/ /media/Projekt\%20sites/Danmap/DANMAP\%20 reports/DANMAP\%202012/Danmap_2012.ashx

30. LozanoC, AspirozC, SáenzY, Ruiz-GarcíaM, Royo-GarcíaG, Gómez-SanzE, et al. Genetic environment and location of the Inu(A) and Inu(B) genes in methicillin-resistant Staphylococcus aureus and other staphylococci of animal and human origin. J Antimicrob Chemother. 2012;67(12):2804-8. DOI: 10.1093/jac/ dks320 PMID: 22899804

31. Statens Serum Institut (SSI). MRSA CC398-epidemiologien I Danmark. [MRSA CC398 epidemiology in Denmark]. Copenhagen: SSI; 2014. [Accessed 30 Oct 2014]. Danish. Available from: http://www.ssi.dk/Aktuelt/Nyhedsbreve/EPINYT/2014/Uge\%2024a\%20-\%202014.aspx

32. DANMAP 2009. Use of antimicrobial agents and occurrence of antimicrobial resistance in bacteria from food animals, food and humans in Denmark. Copenhagen: Statens Serum Institut, Danish Veterinary and Food Administration, Danish Medicines Agency, National Veterinary Institute and National Food Institute, Technical University of Denmark; September 2010. [Accessed 30 Oct 2014]. Available from: http://danmap. org/ /media/Projekt\%2osites/Danmap/DANMAP\%2oreports/ Danmap_2009.ashx

33. on behalf of the CAM Study Group,van RijenMM, Kluytmansvan den BerghMF, VerkadeEJ, Ten HamPB, FeingoldB], KluytmansJA. Lifestyle-associated risk factors for communityacquired methicillin-resistant Staphylococcus aureus carriage in the Netherlands: an exploratory hospital-based case-control study.PLOS ONE. 2013;8(6):e65594. DOI: 10.1371/journal. pone.0065594 PMID: 23840344

34. YuF, ChenZ, LiuC, ZhangX, LinX, ChiS, et al. Prevalence of Staphylococcus aureus carrying Panton-Valentine leukocidin genes among isolates from hospitalised patients in China. Clin Microbiol Infect. 2008;14(4):381-4. DOI: 10.1111/j.14690691.2007.01927.x PMID: 18190580

35. van RijenMM, Van KeulenPH, KluytmansJA. Increase in a Dutch hospital of methicillin-resistant Staphylococcus aureus related to animal farming.Clin Infect Dis. 2008;46(2):261-3. DOI: 10.1086/524672 PMID: 18171259

36. Welinder-OlssonC, Florén-JohanssonK, LarssonL, ObergS, KarlssonL, AhrénC. Infection with Panton-Valentine leukocidinpositive methicillin-resistant Staphylococcus aureus to34.Emerg Infect Dis. 2008;14(8):1271-2. DOI: 10.3201/ eid1408.071427 PMID: 18680653

37. FeingoldBJ, SilbergeldEK, CurrieroFC, van CleefBA, HeckME, KluytmansJA. Livestock density as risk factor for livestockassociated methicillin-resistant Staphylococcus aureus, the Netherlands.Emerg Infect Dis. 2012;18(11):1841-9. DOI: 10.3201/eid1811.111850 PMID: 23092646 